\title{
Editorial
}

\section{Music education: Why bother?}

One of the common questions asked of music educators is some sort of variation on 'music education - why bother?'

'Children and young people', the questioner usually states, 'will get on fine, they'll learn to play instruments and sing if they want to, so why not let the more important subjects in school have the time and money spent on such fripperies?' Usually answering this sort of question requires a deep breath, and a moment to consider what to say so as not to appear too rude. It seems that this question, or one of its near cognates, is increasingly coming to the fore in times of fiscal and social retrenchment. But what do we say, so as to try to silence forever these ill-informed interlocutors? Here in the pages of the British Journal of Music Education we endeavour to present arguments each issue as to why music education is important. But our questioner is unlikely to have read these. In many countries the place of advocacy is high on the list of ways to deal with this, but clearly advocacy alone is not working, else we would not still be hearing such things. In other jurisdictions it is the external, transferable benefits of music education that are promoted, for example, 'music makes you smarter', or 'music makes you better at numeracy'. These are all well and good, but run the danger of something else supplanting music. Some food supplements allegedly make you smarter, and simply going shopping makes you more numerate, so goodbye music, hello lunch and the high street. It seems likely that we as music educators did not become involved with music simply to promote shopping malls? So what can we say to address such questions?

One of our own subject field problems, covered in a number of these BJME editorials passim is that we cannot agree amongst ourselves on what music education actually is (Fautley \& Murphy, 2015b), or what it should contain (Fautley \& Murphy, 2015a). This disagreement is good - it is the sign of a healthy subject domain. It is a truism to note that sport begets competition, and music fosters cooperation, but in neoliberal times, with monetary and societal 'winners' and 'losers' being represented, in a post-truth fashion, as becoming so as a result of their personal 'life choices', this argument may not carry much weight.

But what we can say is what should surely be one of the key reasons for music and musicking, that music is the quintessential human activity (after all it is debatable whether animals intentionally music), and is one that transcends language. As lan Cross observes:

... music may best be thought of as a communicative medium that is optimal for the management of situations of social uncertainty; music is, at root, an excellent means of coordinating social attitudes and behaviours, and can be viewed as complementary to and coextensive in its forms, structures and primitives with speech as an interactive medium. (Cross, 2012)

And surely this is something we need more of in these troubled times? We need to look in to music as a powerful force. Indeed, why else would repressive regimes ban it, or co-opt it for their own twisted ends? 
This is not to say that transfer cannot take place from musical learning, nor that we should downplay it where it does, quite the opposite. After all, if it could be shown that learning music correlates highly with attainment in public examinations, then this would be a significant weapon in our armoury against such comments. Well, it just so happens that the first article in this edition of the $B J M E$, authored by UK researchers and practitioners Susan Hallam and Kevin Rogers, finds that very thing. Indeed, in the piece published in this issue they note that their findings

... showed that the young people playing an instrument showed greater progress and better academic outcomes than those not playing with the greatest impact for those playing the longest.

This is a useful and significant outcome, and gives us a great deal to think about. It also helps go some way towards answering that pesky question we opened with; after all if music not only makes you an all-round better person, but also means that your general attainment is also enhanced, then, as the kids say, 'what's not to like?'

We stay in the UK for our next article, where Stephanie Pitts and Katharine Robinson explore reasons for sustaining and ceasing amateur participation in classical music making. This is another important area for us to consider, as we know that the role of music education for young people often needs to have a through-put that will be continuing well into adulthood. After all, as Bugos puts it,

Contemporary views of music education embrace differences in learning throughout the lifespan and continue to seek equity... (Bugos, 2017 p.xii)

Clearly, we want music education to not be just a short-term affair.

From the UK we move half way around the world to Singapore, where Pamela CostesOnishi describes applications of informal learning procedures by an experienced secondary school music teacher. We are learning more and more about the differences and similarities between formal and informal learning modalities in music education. There are a number of interesting findings in the piece, not least of which is that the

... formal and the informal are complementary rather than distinct and that specifically, for at-risk students, flexibility between the approaches is necessary.

There is much to be learned here, and, as we think increasingly about teaching and learning in a variety of international contexts, articles like this are very helpful.

From Singapore we move to Sweden, for an article by Sven Bjerstedt, who discusses how self-sustaining jazz learning activities can be matched against concepts of 'stolen knowledge', and what the implications are for jazz students and teachers in thinking about how learning to improvise takes place. Our next destination on our international tour takes us to Finland, where Sari Muhonen describes the songcrafting process as reflected on by students some years after they had undertaken the activity. Muhonen also offers us an interesting model of the songcrafting process, which should certainly prove useful to music education students in many countries. 
Finally we return to Sweden for the last stop on our destination board for the issue, where Maria Calissendorff and Haukur Hannesson take us to what is sometimes perceived as the rarified world of the orchestral musician. The authors present us with an overview of research done in this domain, and conclude that

Based on our study of research into training musicians before starting work as professional orchestral players... very little was done specifically on this subject.

This, then presents us with another arena for future research in music education.

The pages of this $B J M E$, as is so often the case, have taken us to a variety of research contexts and national locations in our continued investigations of what is going on at the moment, and it is to be hoped that our questioner cited in the opening of this editorial will find plenty here to give them pause for thought as to why music education is important, and why its continued study occupies so many people in different parts of the globe. It is also to be hoped that as 'bothering' with music education is an important part of our human activity, policy makers and legislators will continue to dig deep into their coffers to find ways of funding its continued presence. For us researchers, clearly there is still so much more we can learn.

MARTIN FAUTLEY

BJME Co-Editor

\section{References}

BUGOS, J. (Ed.) (2017) Contemporary Research in Music Learning across the Lifespan. Abingdon, Routledge. CROSS, I. (2012) 'Music as a social tool'. The Philological Society. Queen Mary, University of London.

FAUTLEY, M. \& MURPHY, R. (2015a) 'Editorial - Difficult questions in music education?' British Journal of Music Education, 32(2), 119-22.

FAUTLEY, M. \& MURPHY, R. (2015b) 'Editorial - What is music education?' British Journal of Music Education, 32(1), 1-4. 